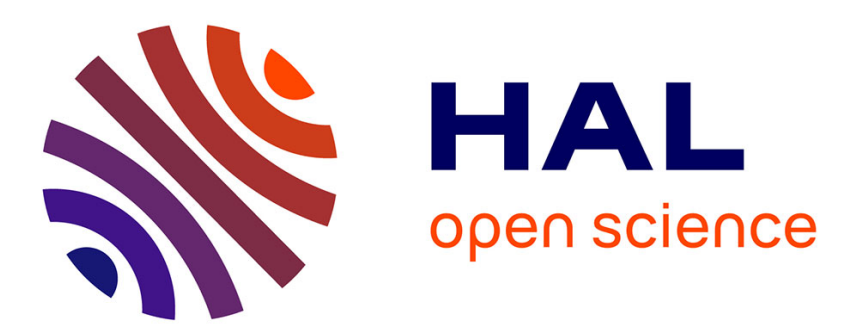

\title{
Influence des contraintes résiduelles de grenaillage sur la microplasticité des solides isotropes
}

\author{
R. Fougères, M. Boivin, A. Parisot, M. Théolier
}

\section{To cite this version:}

R. Fougères, M. Boivin, A. Parisot, M. Théolier. Influence des contraintes résiduelles de grenaillage sur la microplasticité des solides isotropes. Revue de Physique Appliquée, 1979, 14 (8), pp.731-741. 10.1051/rphysap:01979001408073100 . jpa-00244654

\section{HAL Id: jpa-00244654 https://hal.science/jpa-00244654}

Submitted on 1 Jan 1979

HAL is a multi-disciplinary open access archive for the deposit and dissemination of scientific research documents, whether they are published or not. The documents may come from teaching and research institutions in France or abroad, or from public or private research centers.
L'archive ouverte pluridisciplinaire HAL, est destinée au dépôt et à la diffusion de documents scientifiques de niveau recherche, publiés ou non, émanant des établissements d'enseignement et de recherche français ou étrangers, des laboratoires publics ou privés. 


\title{
REVUE DE PHYSIQUE APPLIQUÉE
}

Classification

Physics Abstracts

$62.20 \mathrm{~F}-62.90-81.00$

\section{Influence des contraintes résiduelles de grenaillage sur la microplasticité des solides isotropes}

\author{
R. Fougères $\left({ }^{*}\right)$, M. Boivin $\left({ }^{* *}\right)$, A. Parisot $\left({ }^{*}\right)$ et M. Théolier $\left({ }^{*}\right)$ \\ $\left(^{*}\right)$ Laboratoire de Métallurgie et Traitements thermiques, INSA Lyon, France \\ (**) Laboratoire de Mécanique des Solides, INSA Lyon, France
}

(Reçu le 7 novembre 1978, révisé le 21 mars 1979, accepté le 16 mai 1979)

Résumé. - Nous présentons le calcul de l'influence des contraintes internes sur les propriétés microplastiques d'échantillons cylindriques grenaillés soumis à torsion, présentant un champ de contraintes internes d'extension à cœur, de compression dans les zones périphériques, dues à un écrouissage localisé. Nous avons un bon accord entre les valeurs théoriques et expérimentales et nous montrons que l'influence des contraintes internes de compression sur la microplasticité est compensée pour une large part par l'écrouissage introduit par le grenaillage. Nous montrons également les effets d'un surgrenaillage et d'un chauffage.

\begin{abstract}
A theoretical method for the effect of internal stresses on the microplasticity of isotropic material is applied to the torsion of shot peened cylindrical samples with compressive internal stresses at the surface and extensive ones at the centre due to the local strain-hardening. We obtain theoretical values which are in good agreement with the experimental ones. Strain hardening of peening compensates mainly the effect of compressive internal stresses on plastic microstrain. We put in evidence the effect of a heavy shot-peening and of a heating.
\end{abstract}

1. Introduction. - Les contraintes auxquelles nous nous intéressons sont appelées contraintes internes ou résiduelles, car elles sont présentes dans un solide en l'absence de forces extérieures. D'une manière plus précise [1], [2] le solide a subi un champ de déformations permanentes qui ne vérifie pas les équations de compatibilité de la mécanique du solide. Dans ce cas, le solide doit nécessairement être le siège de déformations additionnelles telles que la déformation résultante soit compatible; le champ de contraintes, associé à cette déformation complémentaire, est un champ autoéquilibré qui est le champ de contraintes internes.

Les causes d'apparition de telles contraintes peuvent être mécaniques, thermiques ou physico-chimiques. Elles se rencontrent fréquemment lors de la mise en forme et des traitements thermiques des métaux et alliages. La détermination expérimentale a été menée principalement par deux types de méthodes. Les unes, non destructives, reposent essentiellement sur la mesure par diffraction des rayons $\mathbf{X}$, de l'évolution des paramètres de maille des solides cristallisés sous l'effet de ces contraintes [3]. Les autres, destructives, procèdent par découpage macroscopique du solide, qui retrouve un nouvel équilibre en se déformant. A partir de ces déformations et de la théorie de l'élasticité, on peut remonter au champ de contraintes internes [4].
Il faut noter que ces deux méthodes intéressent un volume d'échantillon important, en fait macroscopique, et qu'ainsi elles ne prennent en compte que la composante des contraintes internes qui n'est pas en équilibre sur ce volume. Certains auteurs [5] les appellent contraintes internes de premier ordre ou encore contraintes résiduelles. Par la méthode destructive, certains d'entre nous ont pu déterminer à l'intérieur d'échantillons cylindriques des champs de contraintes internes triaxiaux d'origine mécanique ou physico-chimique [6], [7].

La connaissance de ces contraintes est d'un grand intérêt, puisqu'elles se superposent aux contraintes dues aux efforts appliqués. Ce travail consiste en la prise en compte de l'influence de ces contraintes internes sur les propriétés microplastiques des matériaux dans le cas de sollicitations simples. Il présente un intérêt pratique, puisque les valeurs de limite élastique utilisées dans le calcul des structures sont définies dans le domaine microplastique dont l'étendue des déformations rémanentes est comprise entre $10^{-5}$ et quelque $10^{-3}$.

2. Hypothèses et méthode de calcul de la torsion d'un barreau cylindrique circulaire grenaillé. - 2.1 CHAMP DE CONTRAINTES INTERNES DÛ AU GRENAILLAGE. - Le grenaillage consiste à déformer plastiquement la 
périphérie d'un solide par projection de billes d'acier à très grande vitesse. A la surface de cette zone, le solide est demeuré continu et, sur quelques dixièmes de millimètres d'épaisseur, il présente une déformation plastique que l'on peut considérer comme homogène [8]. Le reste du barreau n'est pas affecté. Cette hétérogénéité introduit des contraintes internes de compression à la périphérie du solide et d'extension à cœur. Nous présentons ci-dessous l'évolution du champ de contraintes internes pour le grenaillage normal d'un barreau cylindrique circulaire. Il a été obtenu dans le cadre d'une autre étude, sur un acier à $0,74 \%$ de carbone, trempé et revenu, et dont les conditions précises de préparation des échantillons et d'expérimentation ont été publiées par ailleurs [9].

Le champ de contraintes résiduelles présente une symétrie cylindrique analogue à celle du barreau. Nous n'avons représenté à la figure 1 que les contraintes internes dans une zone de quelques dixièmes de millimètre sous la surface.

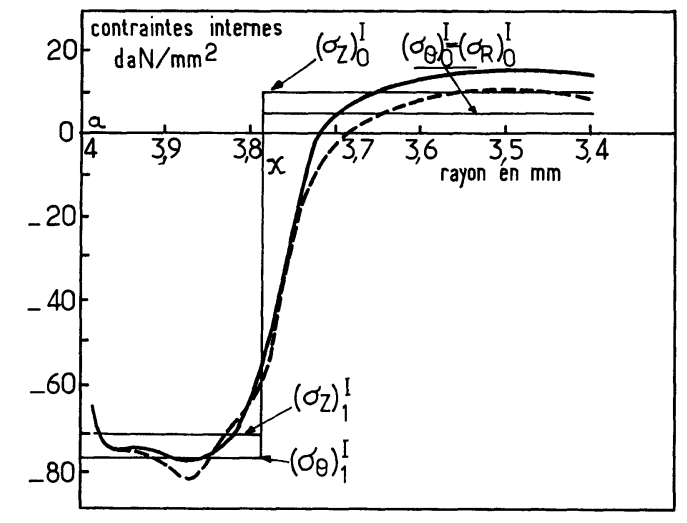

Fig. 1. - Champ de contraintes internes dû au grenaillage normal et sa schématisation pour le calcul.

$$
\begin{aligned}
& \left(\sigma_{z}\right)_{1}^{\mathrm{I}},\left(\sigma_{z}\right)_{0}^{\mathrm{I}}: \text { contrainte longitudinale, } \\
& \left(\sigma_{\theta}\right)_{1}^{\mathrm{I}},\left(\sigma_{\theta}\right)_{0}^{\mathrm{I}}: \text { contrainte circonférentielle, } \\
& \left(\sigma_{r}\right)_{0}^{\mathrm{I}} \quad \text { : contrainte radiale, } \\
& \left(\sigma_{r}\right)_{0}^{\mathrm{I}}=\left(\sigma_{\theta}\right)_{0}^{\mathrm{I}} .
\end{aligned}
$$

[Internal stresses field after average shot-peening and its schematic representation for the calculation.

$$
\begin{gathered}
\left(\sigma_{z}\right)_{1}^{I},\left(\sigma_{z}\right)_{0}^{I}: \text { longitudinal stress, } \\
\left(\sigma_{\theta}\right)_{1}^{I},\left(\sigma_{\theta}\right)_{0}^{I}: \text { circumferential stress, } \\
\left(\sigma_{r}\right)_{0}^{I} \quad: \text { radial stress, } \\
\left.\left(\sigma_{r}\right)_{0}^{I}=\left(\sigma_{\theta}\right)_{0}^{I}\right] .
\end{gathered}
$$

Le barreau cylindrique présente en surface des contraintes internes principales, longitudinales $\sigma_{z}$ et circonférentielles $\sigma_{\theta}$ de compression, proches l'une de l'autre et dont la valeur minimale atteint quelque $-80 \mathrm{daN} / \mathrm{mm}^{2}$. A trois dixièmes de millimètre sous la surface, ces contraintes deviennent positives, atteignent une dizaine de $\mathrm{daN} / \mathrm{mm}^{2}$ et conservent cette valeur jusqu'au centre du barreau. Il existe également une contrainte radiale égale à la contrainte circonférentielle.

Les résultats précédents ont été obtenus pour un seul passage des échantillons sous le jet de la grenaille.

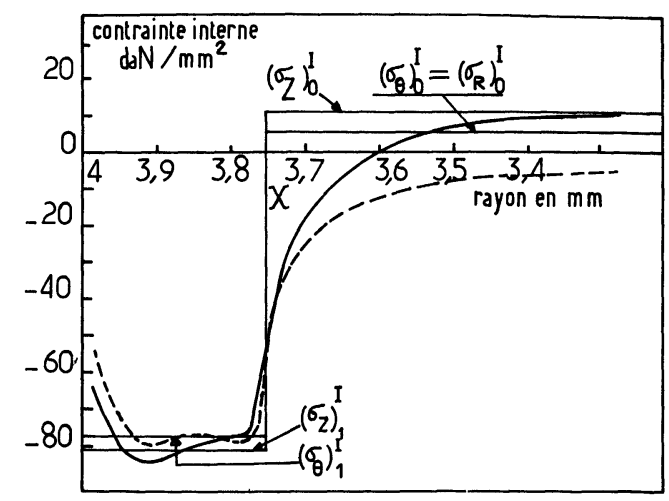

Fig. 2. - Champ de contraintes internes dû au surgrenaillage et sa schématisation pour le calcul.

$$
\begin{aligned}
& \left(\sigma_{z}\right)_{1}^{\mathrm{I}},\left(\sigma_{z}\right)_{0}^{\mathrm{I}}: \text { contrainte longitudinale, } \\
& \left(\sigma_{\theta} \mathrm{I}_{1}^{1},\left(\sigma_{\theta}\right)_{0}^{\mathrm{I}}:\right. \text { contrainte circonferentielle, } \\
& \left(\sigma_{r}\right)_{0}^{\mathrm{I}} \quad \text { : contrainte radiale, } \\
& \left.\left(\sigma_{r}\right)_{0}^{\mathrm{I}}=\left(\sigma_{\theta}\right)_{0}^{\mathrm{I}}\right] .
\end{aligned}
$$

[Internal stresses field after heavy shot-peening and its schematic representation for the calculation.

$$
\begin{aligned}
& \left(\sigma_{z}\right)_{1}^{\mathbb{I}},\left(\sigma_{z}\right)_{0}^{I}: \text { longitudinal stress, } \\
& \left(\sigma_{\theta}\right)_{1}^{\mathrm{I}},\left(\sigma_{\theta}\right)_{0}^{\mathrm{I}}: \text { circumferential stress } \\
& \left(\sigma_{r}\right)_{0}^{\mathrm{I}} \quad: \text { radial stress }, \\
& \left.\left(\sigma_{r}\right)_{0}^{\mathrm{I}}=\left(\sigma_{\theta}\right)_{0}^{\mathrm{I}}\right] .
\end{aligned}
$$

L'influence d'un surgrenaillage correspondant à cinq passages successifs est traduite sur la figure 2 .

On remarque une légère augmentation de la zone en compression ainsi qu'une répartition plus homogène des contraintes résiduelles, tout en conservant des maxima identiques.

Par chauffage, il est possible de réduire ces contraintes résiduelles [9]. Sur la figure 3, nous donnons la répartition de ces contraintes après un chauffage de $220^{\circ}$ pendant $1 \mathrm{~h}$.

On remarque une diminution d'environ $20 \%$ des maxima de contraintes résiduelles.

2.2 HYPOTHÈSES DE CALCUL. - 2.2.1 Schématisation du champ de contraintes internes. - La forme des champs de contraintes internes nous suggère d'utiliser une méthode type méthode des tranches [10] et de schématiser les champs comme l'indiquent les figures 1,2 et 3 , de telle sorte que les conditions d'équilibre soient respectées.

Dans le cas d'un cylindre à section circulaire avec un champ de contraintes internes de symétrie cylindrique, les conditions d'équilibre se résument à [11] :

$$
\int_{S} \sigma_{Z} \mathrm{~d} S=0 \text { et } \int_{0}^{a} \sigma_{\theta} \mathrm{d} r=0
$$

$S$ et $a$ étant respectivement la section droite et le rayon extérieur du barreau.

Nous avons :

- de la surface du barreau située au rayon $a$ à un point défini par le rayon $x$, des contraintes de compression constantes, longitudinales et circonférentielles; 


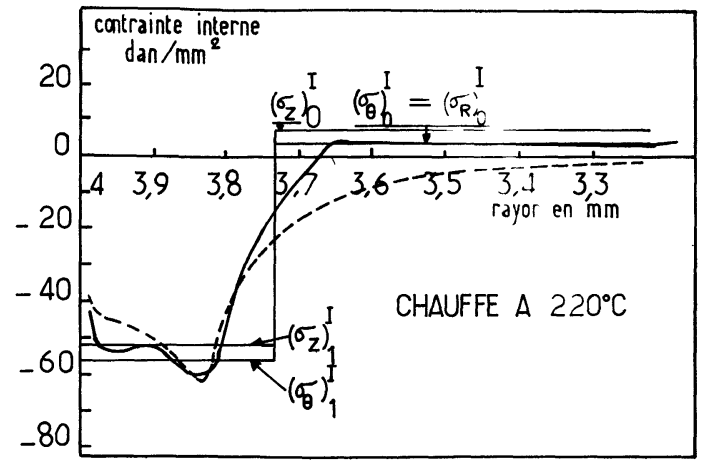

Fig. 3. - Champ de contraintes internes dû au grenaillage normal, suivi d'un chauffage à $220^{\circ} \mathrm{C}$ et sa schématisation pour le calcul. $\left(\sigma_{z}\right)_{1}^{\mathrm{I}},\left(\sigma_{z}\right)_{0}^{\mathrm{I}}:$ contrainte longitudinale ,

$\left(\sigma_{\theta}\right)_{1}^{\mathrm{I}},\left(\sigma_{\theta}\right)_{0}^{\mathrm{I}}:$ contrainte circonférentielle,

$\left(\sigma_{r}\right)_{0}^{1} \quad:$ contrainte radiale ,

$\left(\sigma_{r}\right)_{0}^{\mathbf{I}}=\left(\sigma_{\theta}\right)_{0}^{\mathbf{I}}$.

[Internal stresses field after average shot-peening and heating at $220^{\circ} \mathrm{C}$; schematic representation for the calculation.

$$
\begin{aligned}
& \left(\sigma_{z}\right)_{1}^{\mathrm{I}},\left(\sigma_{z}\right)_{0}^{\mathrm{I}}: \text { longitudinal stress } \\
& \left(\sigma_{\theta} \mathrm{I}_{1}^{\mathrm{I}},\left(\sigma_{\theta}\right)_{0}^{\mathrm{I}}:\right. \text { circumferential stress } \\
& \left(\sigma_{r} \mathrm{I}, \quad \text { radial stress },\right. \\
& \left.\left(\sigma_{r}\right)_{0}^{\mathrm{I}}=\left(\sigma_{\theta}\right)_{0}^{\mathrm{I}}\right]
\end{aligned}
$$

- du rayon $x$ au centre du barreau des contraintes d'extension constantes, longitudinales, circonféren-

\begin{tabular}{|c|c|c|c|c|}
\hline & & $\begin{array}{c}\text { Grenaillage } \\
\text { normal }\end{array}$ & Surgrenaillage & Revenu \\
\hline & & - & - & - \\
\hline$x_{\mathrm{mm}}$ & & 3,79 & 3,75 & 3,73 \\
\hline $\begin{array}{c}\text { Zone } \\
\text { de compression } \\
\text { daN } / \mathrm{mm}^{2}\end{array}$ & $\left\{\begin{array}{l}\sigma_{z} \\
\sigma_{\theta} \\
\sigma_{r}\end{array}\right.$ & $\begin{array}{r}-70 \\
-77 \\
0\end{array}$ & $\begin{array}{r}-77 \\
-81 \\
0\end{array}$ & $\begin{array}{r}-56 \\
-53 \\
0\end{array}$ \\
\hline $\begin{array}{c}\text { Zone } \\
\text { d'extension } \\
\text { daN } / \mathrm{mm}^{2}\end{array}$ & $\left\{\begin{array}{l}\sigma_{z} \\
\sigma_{\theta} \\
\sigma_{r}\end{array}\right.$ & $\begin{array}{r}10 \\
5 \\
5\end{array}$ & $\begin{array}{r}10,7 \\
5,5 \\
5,5\end{array}$ & $\begin{array}{l}8,4 \\
3,8 \\
3,8\end{array}$ \\
\hline
\end{tabular}
tielles et radiales.

Le tableau suivant résume les différentes valeurs de la schématisation.

2.2.2 Loi de comportement plastique. - Le caractère triaxial des contraintes internes nous conduit à utiliser la notion de contrainte uniaxiale équivalente, ce qui suppose le choix d'un critère de plasticité et d'une hypothèse sur l'évolution des contraintes internes au cours de la sollicitation.

Pour les alliages sur lesquels nous appliquerons ce calcul, nous avons pu montrer [12] que, dans le domaine de microplasticité, le critère de Von Misès était vérifié. Cela suppose que l'effet Bauschinger est peu marqué. Nous avons pu vérifier, en effet [13], que le comportement microplastique était indépen- dant du sens de l'application de l'effort. Cela est en accord avec le fait que, pour de faibles déformations plastiques, il n'y a qu'une faible quantité de sources de dislocations activées. En résumé, nous calculerons la contrainte équivalente par la relation suivante déduite du critère de Von Misès :

$\sigma_{\mathrm{eq}}=\frac{1}{\sqrt{2}} \sqrt{\left(\sigma_{11}-\sigma_{22}\right)^{2}+\cdots+6\left(\sigma_{12}^{2}+\cdots\right)}$.

Comme nous considérons de faibles déformations, nous supposerons que le tenseur des contraintes $\sigma_{i j}$ qui intervient dans la relation (1) est tel que :

$$
\sigma_{i j}=\sigma_{i j}^{\mathrm{A}}+\sigma_{i j}^{\mathrm{I}}
$$

avec $\sigma_{i j}^{\mathrm{A}}$ le tenseur des contraintes appliquées,

$\sigma_{i j}^{\mathrm{I}}$ celui des contraintes internes initiales.

Pour la même raison, bien que les trajets de chargement ne soient pas simples, nous utiliserons la théorie intégrée de Hencky, auquel cas nous introduirons l'expression suivante de la déformation équivalente [14], [15], [16], exprimée en fonction des composantes du tenseur des déformations totales,

$\varepsilon_{\mathrm{eq}}=\frac{\sqrt{2}}{3} \sqrt{\left(\varepsilon_{11}-\varepsilon_{22}\right)^{2}+\cdots+6\left(\varepsilon_{12}^{2}+\cdots\right)}$.

2.2.3 Loi d'écrouissage. - Nous supposerons un matériau isotrope, écrouissable, dont, en l'absence de contrainte, la loi de comportement en traction uniaxiale est définie par la figure 4.

Pour :

$-0<\varepsilon_{\mathrm{eq}}<\varepsilon_{\mathrm{s}}\left(\varepsilon_{\mathrm{s}}\right.$ étant la déformation à la limite élastique)

$$
\sigma_{\mathrm{eq}}=3 G \varepsilon_{\mathrm{eq}}
$$

où $G$ est le module d'élasticité transversale.

$$
-\varepsilon_{\mathrm{eq}}>\varepsilon_{\mathrm{s}}
$$

$$
\sigma_{\mathrm{eq}}=3 G \varepsilon_{\mathrm{eq}}+h(e)
$$

où $h(e)$ est une fonction de $e=\varepsilon_{\mathrm{eq}}-\varepsilon_{\mathrm{s}}$.

S'il existe des domaines dans le solide où il y a eu écrouissage préalable, nous supposons que la loi d'écoulement est celle représentée par la courbe B de la figure 4.

\subsubsection{Calcul d'un barreau cylindrique circulaire} soumis à torsion. - Nous supposons qu'il existe une symétrie de révolution et que nous avons $n$ domaines concentriques soumis à des champs de contraintes internes constants. Au cours de la torsion, nous faisons l'hypothèse que tous ces domaines présentent le même angle de rotation unitaire $\theta$ (Fig. 5). Nous verrons plus loin qu'un bon accord entre le calcul et l'expérience pour différents cas de grenaillage justifie cette hypothèse. 


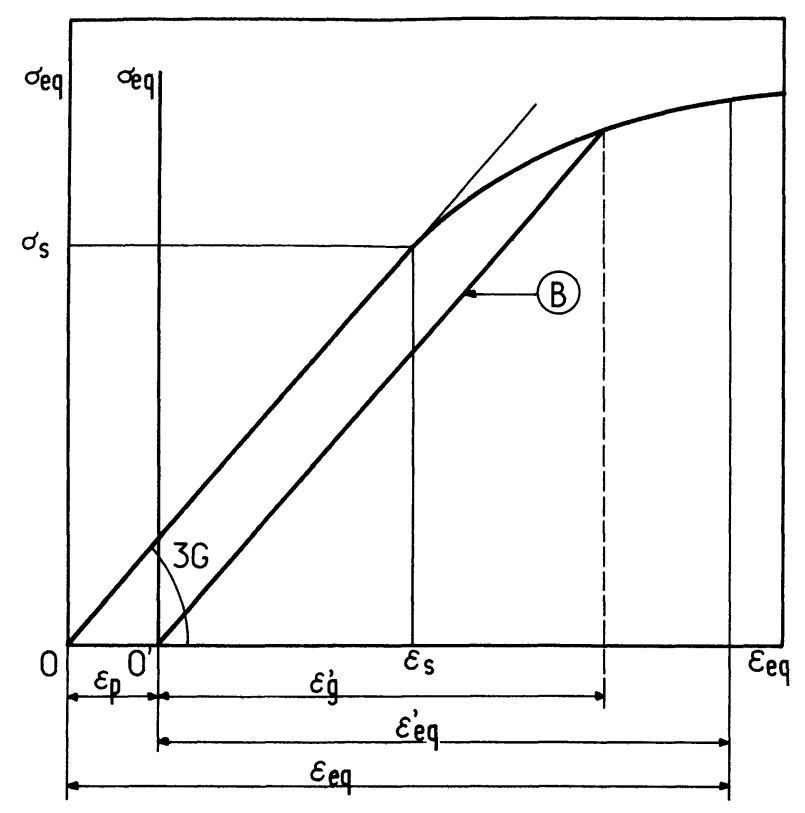

Fig. 4. - Courbe contrainte-déformation équivalente.

[Equivalent stress-strain curve.]

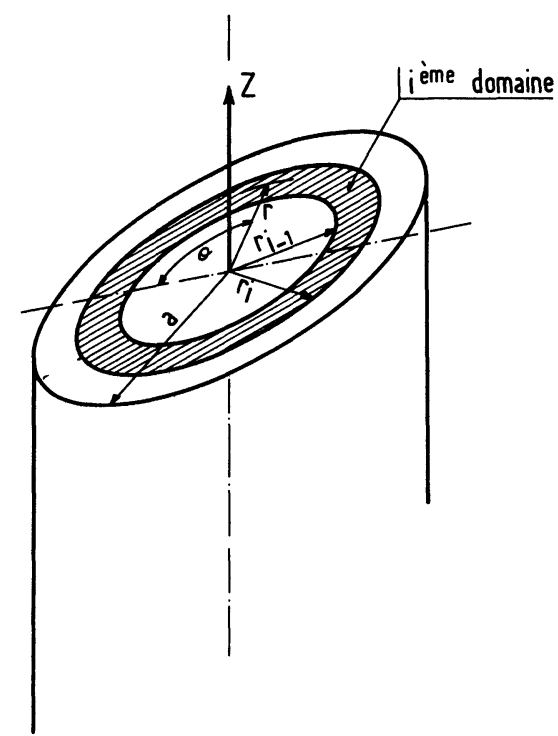

Fig. 5. - Sollicitation en torsion, axes en coordonnées cylindriques.

[Torsional sollicitation in cylindrical coordinates.]

Si $M$ est le moment de torsion, on obtient :

$$
M=\sum_{i=1}^{n} M_{i}(\theta)
$$

avec, pour le tube $i$ :

$$
M_{i}=2 \pi \int_{r_{i-1}}^{r_{i}} \sigma_{\theta z} r^{2} \mathrm{~d} r
$$

où : $r_{i}$ et $r_{i-1}$ sont respectivement les rayons extérieur et intérieur du $i$-ième domaine, $\sigma_{\theta z}$ la contrainte de torsion appliquée et $r$ le rayon courant du barreau.

Si on exprime $\sigma_{\theta z}$ à partir des équations (1), (2) et (3), on obtient, après intégration, l'expression du moment $M_{i}$ en fonction de $\theta$.

En reportant les valeurs de $M_{i}$ dans l'équation (5), on trouve la relation entre $M$ et $\theta$. Pour une valeur du moment $M$ donnée, il est alors possible de calculer la valeur de l'angle de rotation unitaire $\theta$.

3. Résultats obtenus. - Les résultats suivants ont été obtenus sur un acier à $0,74 \%$ de carbone à l'état trempé et revenu.

3.1 DÉTERMINATION DE LA LOI D'ÉCROUISSAGE DU MATÉRIAU. - Des essais de torsion sur tubes très minces de cet acier non grenaillé nous ont fourni la courbe

$$
\sigma_{\theta z}=f\left(\varepsilon_{\theta z}\right) .
$$

Or, le critère de Von Misès nous donne dans ce cas :

et

$$
\begin{aligned}
& \sigma_{\mathrm{eq}}=\sqrt{3} \sigma_{\theta z} \\
& \varepsilon_{\mathrm{eq}}=\frac{2}{\sqrt{3}} \varepsilon_{\theta z} .
\end{aligned}
$$

On en déduit alors la courbe d'écrouissage que l'on met sous la forme (4) :

$$
\sigma_{\mathrm{eq}}=3 G \varepsilon_{\mathrm{eq}}+A_{2}\left(\varepsilon_{\mathrm{eq}}-\varepsilon_{\mathrm{s}}\right)^{2} .
$$

Nous déterminons $A_{2}$ à partir des valeurs expérimentales du domaine microplastique en utilisant une méthode des moindres carrés.

Pour l'acier étudié, nous avons calculé à partir de résultats expérimentaux obtenus à la température de $50^{\circ} \mathrm{C}$ et sur une machine de torsion particulièrement sensible [12] les valeurs suivantes :

$$
\begin{aligned}
& G=7000 \mathrm{daN} / \mathrm{mm}^{2} \\
& A_{2}=-2,92 \times 10^{6} \mathrm{daN} / \mathrm{mm}^{2} \\
& \varepsilon_{\mathrm{s}}=4,07 \times 10^{-3}\left(\text { limite élastique à } 5 \times 10^{-6}\right) . \\
& \text { 3.2 DÉTERMINATION DES RELATIONS } M=f(\theta) .
\end{aligned}
$$
Afin de montrer l'influence des contraintes internes, nous avons procédé au calcul de $\theta$ dans les quatre cas suivants :

- un barreau exempt de contraintes internes, - un barreau présentant un champ de contraintes internes dû à un grenaillage normal,

- un barreau surgrenaillé,

- un barreau grenaillé normalement et ayant subi un chauffage à $220^{\circ} \mathrm{C}$ pendant une heure.

3.2.1 Cylindre sans contrainte interne. - Nous définissons deux domaines selon le rayon du barreau : $0<r<\omega \rightarrow$ domaine élastique (domaine 1); $\omega<r<a \rightarrow$ domaine plastique à la périphérie (domaine 2). 
Dans le domaine 1 , le calcul de $M_{1}$ est immédiat. On a :

$$
M_{1}=\frac{\pi G \omega^{4}}{2} \cdot \theta
$$

$\omega$ est calculé à partir de la relation (2) dans le cas de la torsion simple avec $\varepsilon_{\mathrm{eq}}=\varepsilon_{\mathrm{s}}$, soit :

$$
\omega=\frac{\sqrt{3}}{\theta} \cdot \varepsilon_{\mathrm{s}} \text {. }
$$

Dans le domaine 2, la relation (6) nous donne :

$$
M_{2}=2 \pi \int_{\omega}^{a} \sigma_{\theta z} r^{2} \mathrm{~d} r
$$

Toujours dans le cas de la torsion simple, en exprimant $\sigma_{\theta z}, r$ et $\mathrm{d} r$ à partir des relations (1) et (2), l'expression du couple $M_{2}$ peut se mettre sous la forme :

$M_{2}=\frac{6 \pi}{\theta^{3}} \int_{\varepsilon_{s}}^{\varepsilon_{0 q_{a}}}\left(3 G \varepsilon_{e q}+A_{2}\left(\varepsilon_{e q}-\varepsilon_{\mathrm{s}}\right)^{2}\right) \cdot \varepsilon_{\mathrm{eq}}^{2} \mathrm{~d} \varepsilon_{\mathrm{eq}}$

avec $\varepsilon_{\mathrm{eq} a}=\frac{a \theta}{\sqrt{3}}$, expression tirée de la relation (2).

Après intégration de (9) et addition avec (8), on obtient la valeur de $M=M(\theta)$. Connaissant $M$, il est alors possible de calculer $\theta$ et réciproquement.

L'expression de $M=M(\theta)$ est donnée en annexe.

3.2.2 Cylindre présentant des contraintes internes de grenaillage associées à un écrouissage en surface. L'analyse que nous développons ci-dessous est valable pour tous les cas de grenaillage.

3.2.2.1 Définition des données. - Nous partageons le cylindre en quatre domaines concentriques et nous choisissons un système d'axes cylindriques (Fig. 5).

$* 0<r<\omega$ : comportement élastique.

$$
\begin{aligned}
-\left(\sigma_{i j}\right)^{\mathrm{I}} & =\left(\begin{array}{ccc}
\left(\sigma_{r}\right)_{0} & 0 & 0 \\
0 & \left(\sigma_{\theta}\right)_{0} & 0 \\
0 & 0 & \left(\sigma_{z}\right)_{0}
\end{array}\right) \\
\text { - } \quad\left(\sigma_{i j}\right)^{\mathbf{A}} & =\left(\begin{array}{ccc}
0 & 0 & \sigma_{\theta z} \\
0 & 0 & 0 \\
\sigma_{\theta z} & 0 & 0
\end{array}\right) .
\end{aligned}
$$

Avec la relation (1), la contrainte de cisaillement appliquée dans le domaine 1 est de la forme :

$$
\text { - } \quad\left(\sigma_{\theta z}\right)_{1}=\frac{1}{\sqrt{3}} \sqrt{\sigma_{\text {eq }}^{2}-X_{0}}
$$

où $X_{0}$ est défini par :

$$
\begin{aligned}
X_{0}=\left(\sigma_{z}\right)_{0}^{2}+\left(\sigma_{\theta}\right)_{0}^{2} & +\left(\sigma_{r}\right)_{0}^{2}-\left(\sigma_{z}\right)_{0}\left(\sigma_{\theta}\right)_{0}- \\
& -\left(\sigma_{\theta}\right)_{0}\left(\sigma_{r}\right)_{0}-\left(\sigma_{r}\right)_{0}\left(\sigma_{z}\right)_{0}
\end{aligned}
$$

- pour $r=0$, on a, d'après la relation (2),

$$
\varepsilon_{\mathrm{eq}}=\varepsilon_{0}=\frac{1}{3 G} \sqrt{X_{0}}
$$

$* \omega<r<x$ : comportement plastique.

Les différentes grandeurs sont les mêmes que dans le domaine précédent, seule $\sigma_{\text {eq }}$ a une expression mathématique différente.

$* x<r<a$.

Dans ce domaine, la loi d'écrouissage du matériau est différente en raison d'un écrouissage préalable introduit par le grenaillage. L'un d'entre nous [8] a calculé, dans le cas du grenaillage normal, cette valeur de la déformation plastique en considérant, d'après le théorème de la décharge [15], que les contraintes résiduelles résultent de la différence entre l'état de chargement réel et l'état de chargement fictif. L'état de chargement réel est celui d'un tube, d'épaisseur égale à celle de la zone où les contraintes internes sont de compression, entièrement plastique sous l'effet d'une pression extérieure et d'un effort axial, et dans lequel est emmanché élastiquement un noyau. Le chargement fictif est du même type, mais l'ensemble tube-noyau a un comportement élastique. Cette analyse fait apparaître un champ de contraintes analogue à la schématisation retenue au paragraphe 2.2.1. En ajustant les valeurs des contraintes internes ainsi calculées et celles déterminées expérimentalement, on peut calculer la déformation plastique équivalente introduite. Pour un découpage assez voisin de celui retenu au paragraphe 2.2.1, cela correspond à une déformation plastique équivalente légèrement supérieure à $1 \times 10^{-3}$.

En toute généralité, nous supposerons que cette zone, comprise entre $x$ et $a$, comprend deux domaines :

a) $x<r<v$ : comportement élastique.

$$
\begin{aligned}
& \text { * } \quad\left(\sigma_{i j}\right)^{I}=\left(\begin{array}{ccc}
\left(\sigma_{r}\right)_{1} & 0 & 0 \\
0 & \left(\sigma_{\theta}\right)_{1} & 0 \\
0 & 0 & \left(\sigma_{z}\right)_{1}
\end{array}\right) \\
& \text { * } \quad\left(\sigma_{i j}\right)^{\mathbf{A}}=\left(\begin{array}{ccc}
0 & 0 & \sigma_{\theta z} \\
0 & 0 & 0 \\
\sigma_{\theta z} & 0 & 0
\end{array}\right) \\
& \text { * } \quad\left(\sigma_{\theta z}\right)_{3}=\frac{1}{\sqrt{3}} \sqrt{\sigma_{\text {eq }}^{2}-X_{1}}
\end{aligned}
$$

avec la valeur de $X_{1}$ définie par :

$$
\begin{aligned}
X_{1}=\left(\sigma_{z}\right)_{1}^{2}+\left(\sigma_{\theta}\right)_{1}^{2} & +\left(\sigma_{r}\right)_{1}^{2}-\left(\sigma_{\theta}\right)_{1}\left(\sigma_{z}\right)_{1}- \\
& -\left(\sigma_{\theta}\right)_{1}\left(\sigma_{r}\right)_{1}-\left(\sigma_{r}\right)_{1}\left(\sigma_{z}\right)_{1} .
\end{aligned}
$$

La déformation équivalente due aux contraintes résiduelles est :

$$
\varepsilon_{1}=\frac{1}{3 G} \sqrt{X_{1}}
$$


* La loi d'écrouissage est différente puisque la limite du domaine élastique n'est plus $\varepsilon_{\mathrm{s}}$ mais $\varepsilon_{\mathrm{g}}^{\prime}$, valeur correspondant à l'écrouissage introduit par le grenaillage. La nouvelle loi d'écrouissage est du type

$$
\sigma_{\mathrm{eq}}=3 G \varepsilon_{\mathrm{eq}}^{\prime} \text { avec } 0<\varepsilon_{\mathrm{eq}}^{\prime}<\varepsilon_{\mathrm{g}}^{\prime} \quad \text { (voir Fig. 4). }
$$

b) $v<r<a$ : comportement plastique.

Tous les termes sont identiques à ceux du domaine précédent sauf la loi d'écrouissage qui doit tenir compte de la déformation plastique introduite par le grenaillage.

3.2.2.2 Calcul des différents moments de torsion. - Le moment de torsion $M$ est dans ce cas la somme de quatre moments, soit :

$$
\begin{aligned}
& M= M_{1}+M_{2}+M_{3}+M_{4} \\
& M=2 \pi\left(\int_{0}^{\omega}\left(\sigma_{\theta z}\right)_{1} r^{2} \mathrm{~d} r+\int_{\omega}^{x}\left(\sigma_{\theta z}\right)_{2} r^{2} \mathrm{~d} r+\right. \\
&\left.\quad+\int_{x}^{v}\left(\sigma_{\theta z}\right)_{3} r^{2} \mathrm{~d} r+\int_{v}^{a}\left(\sigma_{\theta z}\right)_{4} r^{2} \mathrm{~d} r\right) .
\end{aligned}
$$

3.2.2.2.1 Calcul de $M_{1}$. - En exprimant $\sigma_{\theta z}, r$ et $\mathrm{d} r$ en fonction $\mathrm{de} \varepsilon_{\mathrm{eq}}$ par combinaison des relations (12), (3), (14), (8) et en sachant que $6 \varepsilon_{\theta z}^{2}=\frac{3}{2} r^{2} \theta^{2}$, où $\theta$ est l'angle de rotation unitaire, on obtient :

$$
M_{1}=\frac{18 \pi \cdot G}{\theta^{3}} \int_{\varepsilon_{0}}^{\varepsilon_{\mathrm{s}}}\left(\varepsilon_{\mathrm{eq}}^{2}-\varepsilon_{0}^{2}\right) \varepsilon_{\mathrm{eq}} \mathrm{d} \varepsilon_{\mathrm{eq}} .
$$

On montre facilement que

$$
M_{1}=\frac{\pi G \omega^{4}}{2} \theta \quad \text { avec } \quad \omega=\frac{\sqrt{3}}{\theta} \sqrt{\varepsilon_{\mathrm{s}}^{2}-\varepsilon_{0}^{2}} .
$$

Ce résultat, identique à celui trouvé en l'absence de contrainte interne, est dû au fait que ce domaine a un comportement élastique.

3.2.2.2.2 Calcul de $M_{2}$. - D'une manière analogue au domaine précédent, $M_{2}$ peut se mettre sous la forme :

$$
\begin{array}{r}
M_{2}=\frac{6 \pi}{\theta^{3}} \int_{\varepsilon_{\mathrm{s}}}^{\varepsilon_{x}} \sqrt{\left(3 G \varepsilon_{\mathrm{eq}}+A_{2}\left(\varepsilon_{\mathrm{eq}}-\varepsilon_{\mathrm{s}}\right)^{2}\right)^{2}-9 G^{2} \varepsilon_{0}^{2}} \times \\
\times \sqrt{\varepsilon_{\mathrm{eq}}^{2}-\varepsilon_{0}^{2}} \cdot \varepsilon_{\mathrm{eq}} \mathrm{d} \varepsilon_{\mathrm{eq}}
\end{array}
$$

avec $\varepsilon_{x}=\sqrt{\varepsilon_{0}^{2}+\frac{1}{3} x^{2} \theta^{2}}$.

Pour intégrer cette expression, nous avons été amenés à procéder à une approximation en considérant que $X_{0}$ et $\varepsilon_{0}$ sont respectivement petits devant $\sigma_{\text {eq }}$ et $\varepsilon_{\text {eq }}$. Compte tenu de la valeur des contraintes résiduelles à cœur, cette approximation est très largement justifiée. Aussi, nous posons

$$
\sqrt{\sigma_{\mathrm{eq}}^{2}-X_{0}} \simeq \sigma_{\mathrm{eq}}-\frac{X_{0}}{2 \sigma_{\mathrm{eq}}}
$$

et

$$
\sqrt{\varepsilon_{\mathrm{eq}}^{2}-\varepsilon_{0}^{2}} \simeq \varepsilon_{\mathrm{eq}}-\frac{\varepsilon_{0}^{2}}{2 \varepsilon_{\mathrm{eq}}} .
$$

Dans ces conditions, la valeur du couple $M_{2}$ est donnée en annexe.

3.2.2.2.3 Calcul de $M_{3}$. - Ce calcul est particulièrement simple puisque la sollicitation a lieu dans le domaine élastique. Aussi, le résultat est immédiat :

$$
\begin{aligned}
M_{3} & =\frac{\pi G\left(v^{4}-x^{4}\right)}{2} \theta \\
v & =\frac{\sqrt{3}}{\theta} \sqrt{\varepsilon_{\mathrm{g}}^{\prime 2}-\varepsilon_{1}^{2}} .
\end{aligned}
$$

3.2.2.2.4 Calcul de $M_{4}$. - On pourrait envisager de calculer la valeur de $M_{4}$ d'une manière analogue à celle utilisée pour $M_{2}$. Il suffirait de remplacer $X_{0}$ par $X_{1}$ et de changer les bornes d'intégration, $\varepsilon_{\mathrm{s}}$ en $\varepsilon_{\mathrm{g}}^{\prime}$ et $\varepsilon_{\mathrm{eq} x}$ en $\varepsilon_{\mathrm{eq} a}$. Il est à remarquer que ce calcul n'est valable que dans l'hypothèse où $\varepsilon_{1}$ est petit devant $\varepsilon_{\mathrm{eq}}$. Pour cette zone, l'approximation n'est pas entièrement justifiée puisque $\varepsilon_{1}$ est de l'ordre de la moitié de la distorsion due au couple de torsion. Aussi, pour calculer $M_{4}$, nous allons procéder différemment en remarquant que, pour la valeur de $\varepsilon_{\text {eq }}$ correspondant à l'écrouissage dû au grenaillage, la pente de la loi d'écrouissage est de l'ordre du dixième de celle à l'origine. Aussi, nous supposerons que cette pente est nulle et que la loi d'écrouissage dans le domaine plastique est de la forme :

$$
\sigma_{\mathrm{eq}}=3 G \varepsilon_{\mathrm{g}}^{\prime} .
$$

Dans ces conditions, le couple $M_{4}$ a pour expression :

$M_{4}=\frac{18 \pi G}{\theta^{3}} \int_{v}^{a} \sqrt{\varepsilon_{\mathrm{g}}^{\prime 2}-\varepsilon_{1}^{2}} \cdot \sqrt{\varepsilon_{\mathrm{eq}}^{2}-\varepsilon_{1}^{2}} \cdot \varepsilon_{\mathrm{eq}} \mathrm{d} \varepsilon_{\mathrm{eq}}$.

On montre facilement que :

$$
M_{4}=\frac{6 \pi G}{3^{3 / 2}}\left(a^{3}-v^{3}\right) \cdot \sqrt{\varepsilon_{\mathrm{g}}^{\prime 2}-\varepsilon_{1}^{2}}
$$

avec $v=\frac{\sqrt{3}}{\theta} \sqrt{\varepsilon_{\mathrm{g}}^{\prime 2}-\varepsilon_{1}^{2}}$.

Lorsque toute la zone comprise entre $x$ et $a$ est plastique, le couple $M_{3}$ s'annule; il subsiste $M_{4}$ qui est indépendant de $\theta$ et dont la valeur est donnée par la relation (27) dans laquelle on fait $v=x$.

Ainsi, par sommation des couples $M_{1}, M_{2}, M_{3}$ et $M_{4}$, on aboutit à la relation générale reliant le couple global $M$ à $\theta$ :

$$
M=M(\theta) .
$$

3.3 APPLICATION NUMÉRIQUE ET COMPARAISON DES VALEURS CALCULÉE ET EXPÉRIMENTALE DU COUPLE. - 
Nous récapitulons ici les différentes valeurs numériques introduites dans les calculs :

$$
G=7000 \mathrm{daN} / \mathrm{mm}^{2} ; \quad \varepsilon_{\mathrm{s}}=4,07 \times 10^{-3} ;
$$$$
A_{2}=-2,92 \times 10^{6} \mathrm{daN} / \mathrm{mm}^{2} ; \quad a=4,00 \mathrm{~mm} \text {. }
$$

Pour les trois cas de grenaillage, les valeurs des contraintes internes et du rayon $x$ sont données au tableau I.

Tous les calculs ont été réalisés sur un calculateur Hewlett-Packard № 10.

3.3.1 Cas du cylindre sans grenaillage. - La figure 6 montre l'évolution du couple calculé en fonction de l'angle de rotation unitaire. Nous avons également reporté sur cette figure les valeurs expérimentales mesurées sur la même machine de torsion que précédemment et à une température de $50^{\circ} \mathrm{C}$. Nous remarquons qu'il existe un excellent accord entre les valeurs théoriques du calcul développé et les valeurs expérimentales.

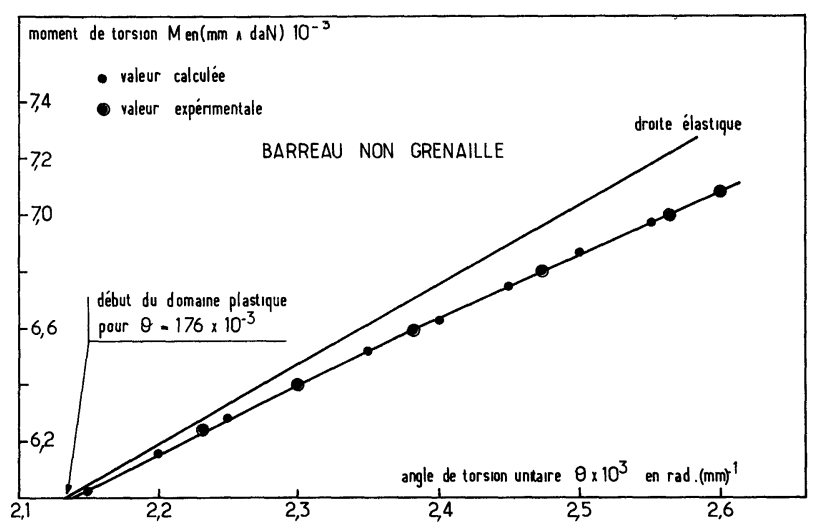

Fig. 6. - Variation du moment de torsion en fonction de l'angle de rotation unitaire, $M=M(\theta)$. Cas du barreau cylindrique non grenaillé. Comparaison entre les valeurs calculée et expérimentale.

[Variation of the twisting moment depending on the angular twist per unit of length, $M=M(\theta)$ in the case of a unpeened cylindrical sample. Comparison between calculated values and experimental ones.]

3.3.2 Cas du cylindre avec grenaillage normal. La figure 7 représente les valeurs expérimentales et les valeurs calculées.

Depuis la fin du domaine élastique jusqu'à B, seule la zone comprise entre les valeurs du rayon $\omega$ et $x$ est plastifiée. Il s'agit de la zone où les contraintes internes sont en extension. A partir d'une valeur de couple voisine de $6,6 \mathrm{~m} \cdot \mathrm{daN}$, la zone extérieure comprise entre $x$ et $a$ commence à se déformer plastiquement. Elle est entièrement plastique au-delà d'une valeur de couple égale à $6,8 \mathrm{~m} \wedge$ daN. Il est à remarquer que les valeurs calculées sont très légèrement supérieures à celles mesurées, la différence étant toutefois significative compte tenu de la précision des mesures. L'écart relatif est plus important lorsque

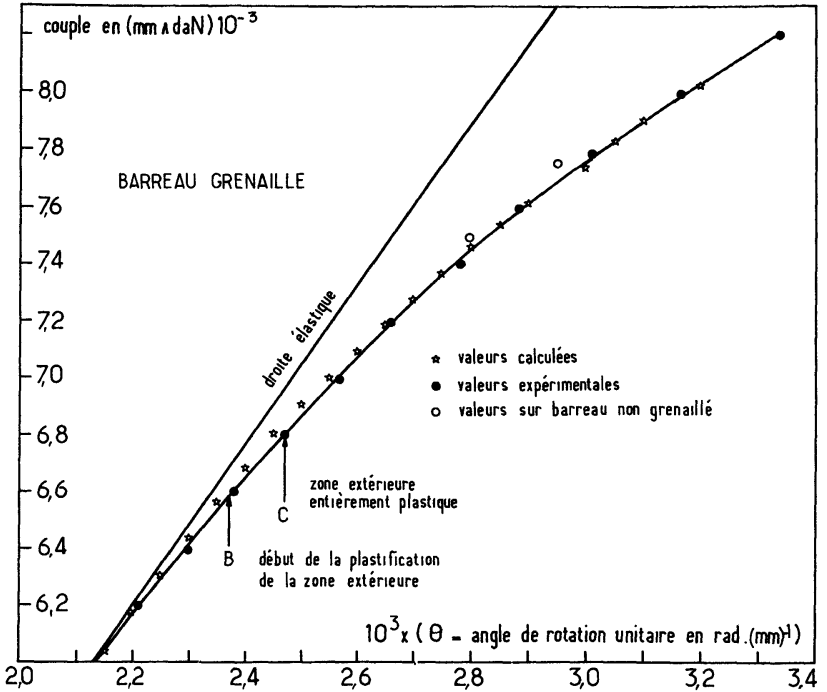

Fig. 7. - Variation du moment de torsion en fonction de l'angle de rotation unitaire $\theta$. Cas du barreau cylindrique grenaillé. Comparaison entre les valeurs calculée et expérimentale.

[Variation of the twisting moment depending on the angular twist per unit of length in the case of a peened cylindrical sample. Comparison between calculated values and experimental ones.]

l'on considère la partie de la déformation comprise entre la droite élastique et la courbe $M=M(\theta)$ (Fig. 7).

$\mathrm{Au}$-delà de $\mathrm{C}$, la zone périphérique est entièrement plastique. On remarque une bonne correspondance entre les valeurs calculées et mesurées. Cet ajustement est assez sensible au choix de la valeur de $\varepsilon_{\mathrm{g}}^{\prime}$ comme en témoigne la courbe de la figure 8 où nous avons porté, pour un angle de torsion $\theta=2,75 \times 10^{-3}$, la valeur du couple en fonction de la limite élastique $\varepsilon_{\mathrm{g}}^{\prime} \mathrm{de}$ la zone affectée par l'écrouissage dû au grenaillage.

Nous constatons effectivement une variation assez rapide du couple avec ce paramètre. La valeur que

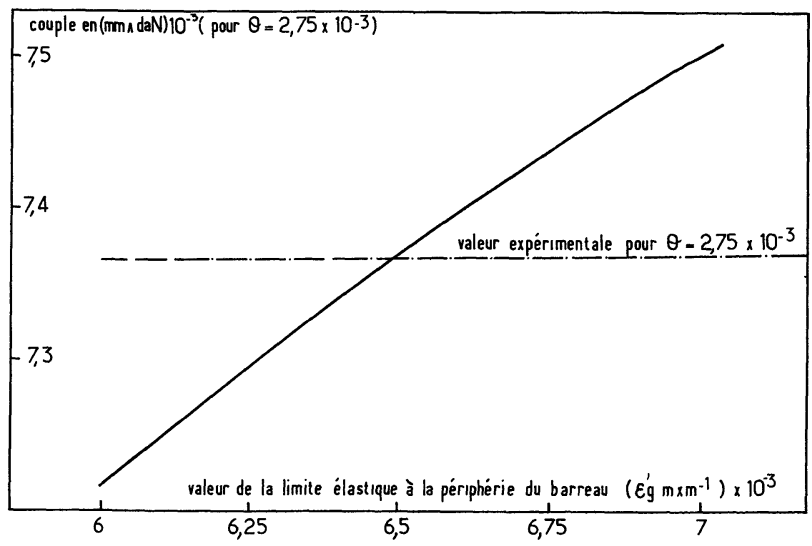

Fig. 8. - Evolution du moment de torsion avec la valeur de la limite élastique $\varepsilon_{\mathrm{g}}^{\prime}$ après écrouissage dû au grenaillage. Angle de torsion $\theta=2,75^{2} \times 10^{-3}$.

[Variation of the twisting moment depending on the value of elasticlimit, $\varepsilon_{g}^{\prime}$, after the strain hardening due to the shot-peening. Angular twisting per unit of length $\theta=2.75 \times 10^{-3}$.] 
nous avons retenue est de $6,5 \times 10^{-3}$, celle pour laquelle le couple calculé est égal à celui mesuré. Cette valeur correspond à une déformation plastique introduite par grenaillage de l'ordre de $1,3 \times 10^{-3}$. Elle est tout à fait comparable à celle calculée par l'un d'entre nous, comme nous l'avons indiqué au paragraphe 3.2.2.

Enfin, sur la figure 7, nous avons reporté également quelques valeurs du barreau non grenaillé. Nous constatons que le grenaillage a un léger effet sur la déformation plastique par torsion.

3.3.3 Cas du cylindre surgrenaillé. - Sur la figure 9, nous avons reporté les valeurs expérimentales et calculées. Nous remarquons à nouveau un excellent accord entre ces valeurs avec, cependant, la même remarque que précédemment pour les faibles déformations plastiques. Il est à remarquer également que la valeur de $\varepsilon_{\mathrm{g}}^{\prime}$ est la même que dans le cas du grenaillé normal. Cela indique qu'un surgrenaillage n'introduit pas un écrouissage plus élevé, mais qu'il étend le domaine affecté ( $x=3,73$ pour le surgrenaillé contre $x=3,79$ pour le grenaillé normal).

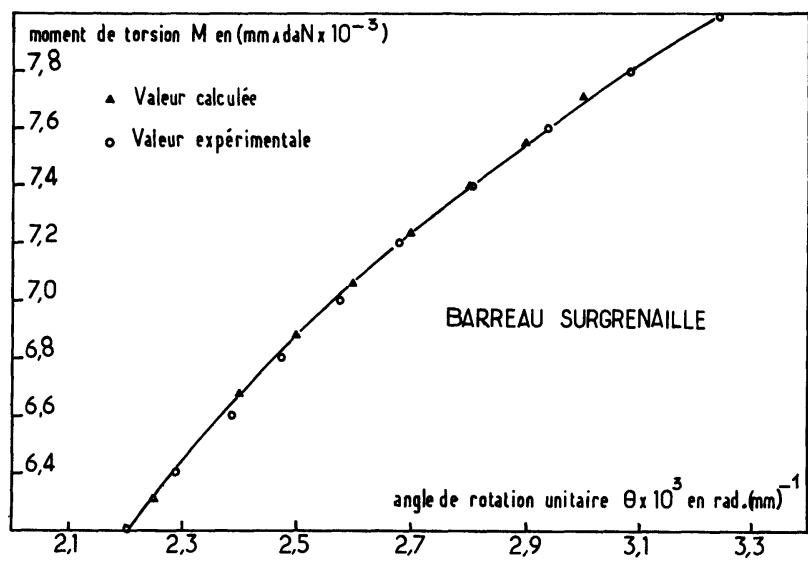

Fig. 9. - Variation du moment de torsion en fonction de l'angle de rotation unitaire, $M=M(\theta)$. Cas du barreau cylindrique surgrenaillé. Comparaison entre les valeurs calculées et expérimentales.

[Variation of the twisting moment depending on the angular twist per unit of length, $M=M(\theta)$ in the case of heavy peened cylindrical sample. Comparison between calculated values and experimental ones.]

3.3.4 Cas du cylindre grenaillé normalement et chauffé à $220^{\circ} \mathrm{C}$. - Sur la figure 10 , on remarque que, dans ce cas, l'accord entre les valeurs théoriques et expérimentales est excellent dans tout le domaine de déformation plastique. Il faut noter que la valeur de $\varepsilon_{\mathrm{g}}^{\prime}$ qui caractérise l'écrouissage dans les zones périphériques est égale à $6,15 \times 10^{-3}$, valeur plus faible qu'après grenaillage.

3.4 ANALYSE DES RÉSUltatS. - - Afin d'expliquer le faible écart observé entre les valeurs théoriques et expérimentales au début de la déformation plastique, plusieurs hypothèses peuvent être émises :

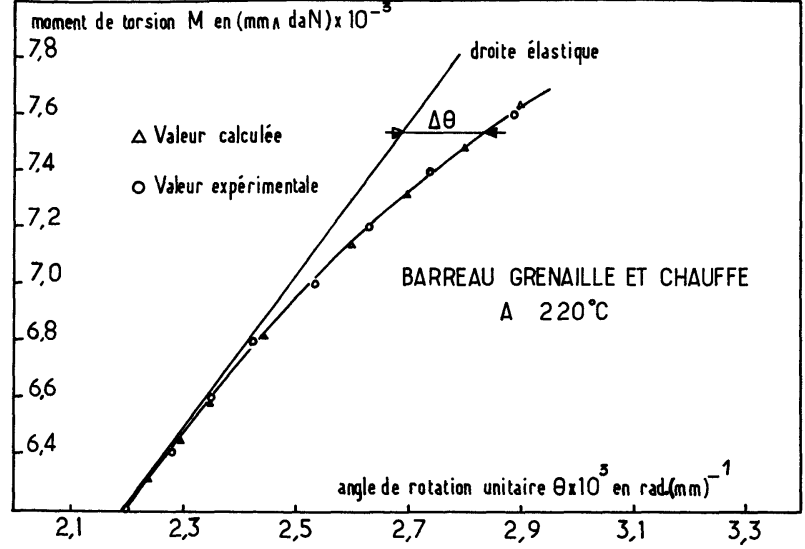

Fig. 10. - Variation du moment de torsion en fonction de l'angle de rotation unitaire, $M=M(\theta)$. Cas du barreau cylindrique grenaillé normalement et chauffé à $200^{\circ} \mathrm{C}$. Comparaison entre les valeurs calculées et expérimentales.

[Variation of the twisting moment depending on the angular twist per unit of length, $M=M(\theta)$ in the case of average peened cylindrical sample, heated at $220^{\circ} \mathrm{C}$. Comparison between calculated values and experimental ones.]

- Calcul effectué avec la théorie intégrée alors qu'il est bien connu que la théorie différentielle d'emploi plus compliqué donne des résultats plus exacts.

- Schématisation du champ de contraintes internes avec une discontinuité pour le rayon $x$ qui s'écarte des courbes de contraintes réelles.

- L'écrouissage dû au grenaillage peut introduire des dislocations libres susceptibles de faire apparaître une composante élastique non linéaire de la déformation, relativement importante pour les faibles couples et que le modèle présenté ne prend pas en compte.

L'excellent accord observé en tout point pour l'échantillon grenaillé, puis chauffé à $200^{\circ} \mathrm{C}$, semble suggérer que la dernière hypothèse est susceptible d'être retenue. En effet, un tel vieillissement peut provoquer un piégeage des dislocations libres par les atomes de carbone en insertion dans la ferrite.

- Afin de montrer l'effet respectif d'un grenaillage, d'un surgrenaillage et d'un chauffage, nous avons porté sur la figure 11 les écarts $\Delta \theta$ entre la droite élastique et la déformation des barreaux grenaillés. $\Delta \theta$ est défini sur la figure 10 . Nous voyons apparaître l'effet complexe de l'écrouissage et des contraintes internes.

Dans le cas du grenaillé normal, il est évident que l'effet des contraintes internes de compression en surface est largement compensé par l'écrouissage introduit par le grenaillage dans cette même zone. Sur la figure 7 , on remarque en effet que la valeur de l'angle de rotation unitaire $\theta$ pour laquelle le tube extérieur $(x<r<a)$ est entièrement plastique est égale à $2,45 \times 10^{-3}$. Dans l'hypothèse où l'on ne tient pas compte de l'écrouissage et en supposant la même loi d'écrouissage en tout point, on peut calculer, à partir de la relation (2), la valeur de $\theta$ pour laquelle il $\mathrm{y}$ a plastification complète, en faisant $\varepsilon_{\mathrm{eq}}=\varepsilon_{\mathrm{s}}$. 


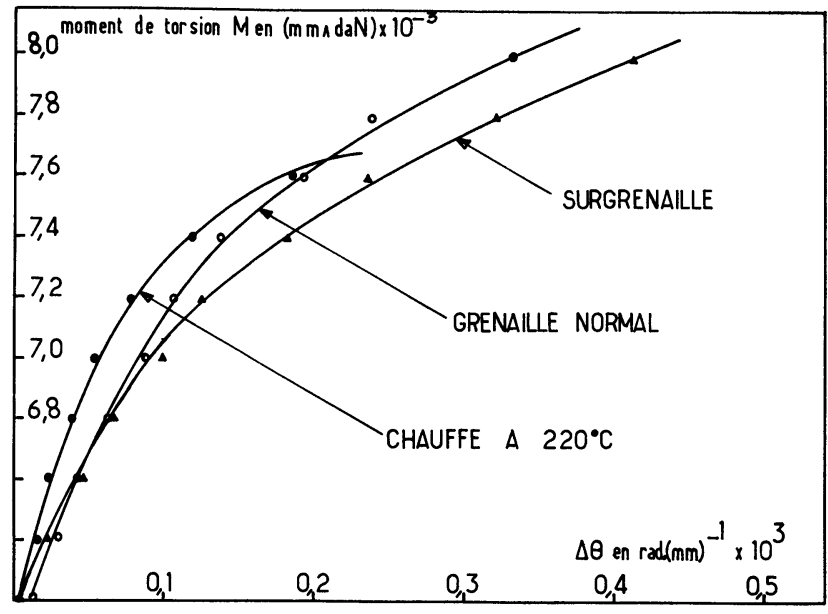

Fig. 11. - Variation dans le domaine plastique des accroissements de l'angle de rotation unitaire $\Delta \theta$ en fonction du couple de torsion.

[Variation of the increases of the angular twist per unit of length, $\Delta \theta$ depending on the twisting moment.]

On trouve alors $\theta=1,6 \times 10^{-3}$. La comparaison de cés deux valeurs indique que la valeur de déformation microplastique calculée en négligeant l'écrouissage serait plus élevée, puisque la plastification apparaîtrait pour des déformations plus faibles que la valeur mesurée expérimentalement.

Le surgrenaillage a pour effet d'augmenter la déformation microplastique, notamment pour les valeurs de couples élevées correspondant à l'entière plastification de la zone extérieure. Cette augmentation est vraisemblablement en relation avec l'accroissement de la zone affectée par les contraintes internes de compression, ainsi qu'avec le niveau de contrainte interne d'extension légèrement plus élevé, pour que globalement, sur l'ensemble de l'échantillon, le champ de contraintes internes soit en équilibre.

La diminution du champ de contraintes internes observé après chauffage indique qu'il est en relation avec l'abaissement de l'écrouissage. Cette remarque suggère que l'approche que nous proposons peut permettre de déterminer non seulement l'écrouissage dû au grenaillage, mais son évolution lors d'un chauffage, si l'on connaît le champ de contraintes internes et la courbe de torsion des échantillons grenaillés.

Les exemples que nous venons de traiter mettent en évidence un aspect qui nous semble important dans la prise en compte des contraintes résiduelles : en effet, ce type de calcul doit impérativement considérer, pour chaque zone du solide, les points sur la courbe contrainte-déformation équivalente, représentatifs des causes qui ont donné naissance à ces contraintes. Un calcul, qui tiendrait compte des contraintes internes, en supposant que l'échantillon présente une loi d'écrouissage en tout point identique, pourrait conduire à des résultats erronés.

En résumé, il nous semble qu'il y ait deux démarches pour le calcul de l'influence des contraintes résiduelles sur la microplasticité des solides :

- Lors du calcul prévisionnel des contraintes internes, on est amené à résoudre le problème complet de plasticité qui aboutit au champ de contraintes internes. On connaît alors l'état de chargement en chaque point du matériau au moment où des efforts extérieurs sont appliqués et le calcul de l'influence des contraintes internes peut alors être effectué.

- L'autre démarche consiste à calculer l'influence des contraintes internes obtenues expérimentalement après avoir déterminé l'évolution de l'écrouissage. On peut connaître expérimentalement cette évolution sur des parties élémentaires de l'échantillon obtenues par découpage, de telle sorte que les contraintes internes peuvent être négligées. Si la forme du champ de contraintes ne permet pas d'obtenir des éléments présentant une telle caractéristique, par exemple pour un champ de contraintes internes à fort gradient, il est alors possible d'en connaitre l'influence sur un essai simple et de remonter à la loi d'écrouissage [12].

Nous pensons que ces deux méthodes, la première plus théorique et la deuxième présentant un caractère expérimental plus affirmé, ne sont pas à opposer, mais qu'au contraire elles sont complémentaires dans l'étude complexe des contraintes internes et de leur influence.

4. Conclusion. - Ce travail porte sur les contraintes internes dont l'origine se situe dans les incompatibilités de déformation plastique qui apparaissent lors de sollicitations mécaniques ou thermiques ou encore lors de transformations physico-chimiques. Nous présentons le calcul de l'influence de ces contraintes internes de grenaillage sur le domaine microplastique des métaux et alliages dont l'amplitude de déformation est compris entre $10^{-5}$ et quelque $10^{-3}$.

Nous développons le calcul de la torsion de barreau cylindrique dans trois cas de grenaillage : un grenaillage normal, un surgrenaillage, un grenaillage normal suivi d'un chauffage.

La forme des champs de contraintes résiduelles nous suggère d'utiliser une méthode de calcul type méthode des tranches et alors de découper le barreau en domaines concentriques soumis à des états de contraintes résiduelles constants d'extension au cœur du barreau et de compression en surface, ces dernières étant associées à un écrouissage dû au grenaillage. Pour un angle de rotation unitaire, $\theta$, supposé uniforme pour les différents domaines, nous calculons les moments correspondants. La somme $M$ de ces moments nous permet d'établir la relation $M=f(\theta)$ pour le barreau complet.

L'étude des trois cas considérés montre qu'il existe un excellent accord entre les valeurs calculées et les valeurs expérimentales.

Le calcul indique que les effets des contraintes internes sont largement compensés par l'écrouissage 
et qu'un surgrenaillage augmente la microplasticité des barreaux en étendant la zone écrouie, bien que la valeur de l'écrouissage reste sensiblement identique à celle du barreau grenaillé normalement. Par contre, lors d'un chauffage, il y a une diminution de l'écrouissage qui conduit à un abaissement de contraintes internes.

En résumé, notre étude indique qu'une méthode de calcul de l'influence des contraintes internes doit obligatoirement intégrer, pour chaque zone du solide, la connaissance sur la courbe contrainte-déformation équivalente du point représentatif du chargement qui a donné naissance au champ de contraintes internes considéré.

\section{Annexe}

1. CAS DU CYLINDRE SANS CONTRAINTE INTERNE.

- Domaine élastique : $0<r<\omega$.

$$
M_{1}=\frac{\pi G \omega^{4} \theta}{2} \text { avec } \omega=\frac{\sqrt{3}}{\theta} \cdot \varepsilon_{\mathrm{s}} \text {. }
$$

- Domaine plastique : $\omega<r<a$.

$$
\begin{gathered}
M_{2}=\frac{6 \pi}{\theta^{3}} \int_{\varepsilon_{\mathrm{s}}}^{\varepsilon_{\mathrm{eq} a}}\left(3 G \varepsilon_{\mathrm{eq}}+A_{2}\left(\varepsilon_{\mathrm{eq}}-\varepsilon_{\mathrm{s}}\right)^{2}\right) \cdot \varepsilon_{\mathrm{eq}}^{2} \mathrm{~d} \varepsilon_{\mathrm{eq}} \\
M_{2}=\frac{6 \pi}{\theta^{3}}\left(\frac{A_{2}}{5}\left(\varepsilon_{\mathrm{eq}_{a}}^{5}-\varepsilon_{\mathrm{s}}^{5}\right)+\frac{3 G-2 \varepsilon_{\mathrm{s}} A_{2}}{4} \times\right. \\
\left.\times\left(\varepsilon_{\mathrm{eq} a}^{4}-\varepsilon_{\mathrm{s}}^{4}\right)+\frac{A_{2} \varepsilon_{\mathrm{s}}^{2}}{3}\left(\varepsilon_{\mathrm{eq}_{a}}^{3}-\varepsilon_{\mathrm{s}}^{3}\right)\right) \\
M=M(\theta)=M_{1}+M_{2} .
\end{gathered}
$$

2. CAS DU CYLINDRE AVEC CONTRAINTE INTERNE ET ÉCROUISSAGE.

- Domaine élastique : $0<r<\omega$.

$$
M_{1}=\frac{\pi G \omega^{4} \theta}{2} \text { avec } \omega=\frac{\sqrt{3}}{\theta} \sqrt{\varepsilon_{\mathrm{s}}^{2}-\varepsilon_{0}^{2}} \text {. }
$$

- Domaine plastique : $\omega<r<x$.

$$
M_{2}=\frac{6 \pi}{\theta^{3}} \int_{\varepsilon_{\mathrm{s}}}^{\varepsilon_{x}} \sqrt{\sigma_{\text {eq }}^{2}-X_{0}} \cdot \sqrt{\varepsilon_{\text {eq }}^{2}-\varepsilon_{0}^{2}} \cdot \varepsilon_{\text {eq }} \mathrm{d} \varepsilon_{\text {eq }}
$$

avec

$$
\varepsilon_{0}=\frac{1}{3 G} \sqrt{X_{0}} \text { et } \sigma_{\mathrm{eq}}=3 G \varepsilon_{\mathrm{eq}}+A_{2}\left(\varepsilon_{\mathrm{eq}}-\varepsilon_{\mathrm{s}}\right)^{2}
$$

en posant

$$
\sqrt{\varepsilon_{\mathrm{eq}}^{2}-\varepsilon_{0}^{2}} \simeq \varepsilon_{\mathrm{eq}}-\frac{\varepsilon_{0}^{2}}{2 \varepsilon_{\mathrm{eq}}}
$$

et

$$
\sqrt{\sigma_{\mathrm{eq}}^{2}-X_{0}} \simeq \sigma_{\mathrm{eq}}-\frac{X_{0}}{2 \sigma_{\mathrm{eq}}}
$$

On a

$$
M_{2}=\frac{6 \pi}{\theta^{3}}\left(I_{1}+I_{2}+I_{3}+I_{4}\right)
$$

avec

$$
\begin{aligned}
& I_{1}=\frac{A_{2}}{5} \cdot\left(\varepsilon_{\mathrm{eq}_{x}}^{5}-\varepsilon_{\mathrm{s}}^{5}\right)+\frac{3 G-2 \varepsilon_{\mathrm{s}} A_{2}}{4}\left(\varepsilon_{\mathrm{eq}_{x}}^{4}-\varepsilon_{\mathrm{s}}^{4}\right)+\frac{A_{2} \varepsilon_{\mathrm{s}}^{2}}{3}\left(\varepsilon_{\mathrm{eq}_{x}}^{3}-\varepsilon_{\mathrm{s}}^{3}\right) \\
& I_{2}=-\frac{\varepsilon_{0}^{2}}{2}\left(\frac{A_{2}}{3}\left(\varepsilon_{\mathrm{eq}_{x}}^{3}-\varepsilon_{\mathrm{s}}^{3}\right)+\frac{3 G-2 \varepsilon_{\mathrm{s}} A_{2}}{2}\left(\varepsilon_{\mathrm{eq}_{x}}^{2}-\varepsilon_{\mathrm{s}}^{2}\right)+A_{2} \varepsilon_{\mathrm{s}}^{2}\left(\varepsilon_{\mathrm{eq}_{x}}-\varepsilon_{\mathrm{s}}\right)\right) \\
& I_{3}=-\frac{9 G^{2} \varepsilon_{0}^{2}}{2}\left(\frac{\varepsilon_{\mathrm{eq}_{x}}-\varepsilon_{\mathrm{s}}}{A_{2}}-\frac{3 G-2 \varepsilon_{\mathrm{s}} A_{2}}{2 A_{2}^{2}} \ln \frac{A_{2} \varepsilon_{\mathrm{eq}_{x}}^{2}+\varepsilon_{\mathrm{eq}_{a}}\left(3 G-2 \varepsilon_{\mathrm{s}} A_{2}\right)+A_{2} \varepsilon_{\mathrm{s}}^{2}}{3 G \varepsilon_{\mathrm{s}}}+\right. \\
& \left.+\frac{\left(3 G-2 \varepsilon_{\mathrm{s}} A_{2}\right)^{2}-2 A_{2} \varepsilon_{\mathrm{s}}^{2}}{2 A_{2}^{2} \sqrt{9 G^{2}-12 G \varepsilon_{\mathrm{s}} A_{2}}} \ln \left|\frac{\frac{2 A_{2} \varepsilon_{\mathrm{eq} x}+3 G-2 \varepsilon_{\mathrm{s}} A_{2}-\sqrt{9 G^{2}-12 G \varepsilon_{\mathrm{s}} A_{2}}}{2 A_{2} \varepsilon_{\mathrm{eq} x}+3 G-2 \varepsilon_{\mathrm{s}} A_{2}+\sqrt{9 G^{2}-12 G \varepsilon_{\mathrm{s}} A_{2}}}}{\frac{3 G-\sqrt{9 G^{2}-12 G \varepsilon_{\mathrm{s}} A_{2}}}{3 G+\sqrt{9 G^{2}-12 G \varepsilon_{\mathrm{s}} A_{2}}}}\right|\right) \\
& I_{4}=\frac{9 G^{2} \varepsilon_{0}^{4}}{4} \frac{1}{\sqrt{9 G^{2}-12 G \varepsilon_{\mathrm{s}} A_{2}}} \ln \left|\frac{\frac{2 A_{2} \varepsilon_{\mathrm{eq}}+3 G-2 \varepsilon_{\mathrm{s}} A_{2}-\sqrt{9 G^{2}-12 G \varepsilon_{\mathrm{s}} A_{2}}}{2 A_{2} \varepsilon_{\mathrm{eq}}+3 G-2 \varepsilon_{\mathrm{s}} A_{2}+\sqrt{9 G^{2}-12 G \varepsilon_{\mathrm{s}} A_{2}}}}{\frac{3 G-\sqrt{9 G^{2}-12 G \varepsilon_{\mathrm{s}} A_{2}}}{3 G+\sqrt{9 G^{2}-12 G \varepsilon_{\mathrm{s}} A_{2}}}}\right|
\end{aligned}
$$


- Domaine pour $x<r<a$.

$* x<r<a$ : domaine élastique.

$$
M_{3}=\frac{\pi G \theta}{2}\left(a^{4}-x^{4}\right)
$$

$* x<r<v$ : domaine élastique et $v<r<a$ : domaine plastique :

$$
M_{3}=\frac{\pi G \theta}{2}\left(v^{4}-x^{4}\right) \text { avec } v=\frac{\sqrt{3}}{\theta} \sqrt{\varepsilon_{\mathrm{g}}^{\prime 2}-\varepsilon_{1}^{2}}
$$

$$
M_{4}=\frac{6 \pi G}{3^{3 / 2}} \cdot \sqrt{\varepsilon_{\mathrm{g}}^{\prime 2}-\varepsilon_{1}^{2}} \cdot\left(a^{3}-v^{3}\right)
$$

avec $v$ la même valeur que ci-dessus.

$* x<r<a$ : domaine plastique.

$$
\begin{aligned}
M_{4} & =\frac{6 \pi G}{3^{3 / 2}} \sqrt{\varepsilon_{\mathrm{g}}^{\prime 2}-\varepsilon_{1}^{2}} \cdot\left(a^{3}-x^{3}\right) \\
M & =M(\theta)=M_{1}+M_{2}+\left(M_{3} \text { ou } M_{3}+M_{4} \text { ou } M_{4}\right) .
\end{aligned}
$$

\section{Bibliographie}

[1] Adda, Y., Dupouy, J.-M., Philibert, J., Quéré, Y., Eléments de métallurgie physique, Tome 1 : rappels. Ed. Commissariat à l'Energie atomique, Saclay (1976).

[2] ZaOUI, A., Les différents types de contraintes internes et leur origine, Séminaire sur les contraintes internes des 16 et 17 novembre 1978, Société Française de Métallurgie Section Sud-Est, Centre de Recherches de Voreppe, Aluminium Péchiney.

[3] GUINIER, A., Théorie et technique de la radiocristallographie (Dunod éditeur, Paris) 1956.

[4] SACHS, G., Nachweiss ennerer Spannungen in Stangen und Rohren, Z. Metal. 9 (1927) 352.

[5] Macherauch, E., Wohlfart, H. et Volfstieg, V., H.T.M. (1973) 28.

[6] Vannes, A., Fougères, R. et Théolier, M., Mét.-Corros. Ind. 578 (1973) 341.

[7] Parisot, A., Fougères, R. et ThÉolier, M., Mét.-Corros. Ind. 602 (1975) 340.

[8] Parisot, A., Détermination et emploi prévisionnel de lois élémentaires de fluage à basse température, Thèse d'Ingénieur-Docteur, Lyon (14 mars 1977).
[9] Parisot, A., Fougères, R., Vannes, A. et ThÉolier, M., Mém. Sci. Rev. Métall. (1977).

[10] Baque, P., D’Escatha, Y., Felder, E. et Hyafil, J., Mise en forme des métaux; calculs par la plasticité, Tome 1 (Dunod) 1973

[11] Pomey, J., Goutel, F. et Abel, L., Mét.-Corros. Ind. 314 (1951) 356.

[12] Fougères, R. et THÉolier, M., Sur le comportement dissymétrique en traction et en compression des martensites d'acier au carbone, C. R. Hebd. Séan. Acad. Sci. $281 \mathrm{C}$ (1976) 909-911.

[13] Fougères, R. et ThÉolier, M., Mém. Sci. Rev. Métall. LXXI, 3 (1974) 169-178.

[14] Borvin, M. et Bahuaud, J., Cours de Mécanique des Solides, Département de Génie Mécanique Construction, INSA Lyon.

[15] ILIOUCHINe, Plasticité (Eyrolles éditeur, Paris) 1956.

[16] Hill, R., The mathematical theory of plasticity (Oxford University Press) 1964. 